\title{
Non-locally compact Polish groups and two-sided translates of open sets
}

by

Maciej Malicki (Urbana, IL, and Warszawa)

\begin{abstract}
This paper is devoted to the following question. Suppose that a Polish group $G$ has the property that some non-empty open subset $U$ is covered by finitely many two-sided translates of every other non-empty open subset of $G$. Is then $G$ necessarily locally compact? Polish groups which do not have the above property are called strongly non-locally compact. We characterize strongly non-locally compact Polish subgroups of $S_{\infty}$ in terms of group actions, and prove that certain natural classes of non-locally compact Polish groups are strongly non-locally compact. Next, we discuss applications of these results to the theory of left Haar null sets. Finally, we show that Polish groups such as the isometry group of the Urysohn space and the unitary group of the separable Hilbert space are strongly non-locally compact.
\end{abstract}

1. Introduction. For a group $G$, subsets of $G$ of the form $f U g$, where $U \subseteq G, f, g \in G$, are called two-sided translates of $U$. Consider the following theorem:

Theorem 1 (Solecki, Uspenskij). A Polish group $G$ is not compact if and only if there exists an open neighborhood $U$ of the identity such that no finite family of two-sided translates of $U$ covers $G$.

Actually, Solecki's result (see [11]) concerns Polish groups only, whereas Uspenskij's theorem (see [15]) is formulated in the general setting. The proof of Theorem 1 is not immediate, essentially because it is not true in general that Polish groups admit a compatible metric with respect to which all two-sided translates of sets $U$ of small diameter have small diameter.

It is natural to ask whether non-locally compact Polish groups can also be characterized in terms of two-sided translates of neighborhoods of the identity. Suppose that a Polish group $G$ admits a non-empty open subset $U$ which is covered by finitely many two-sided translates of every other non-empty open subset of $G$. Is then $G$ necessarily locally compact?

2000 Mathematics Subject Classification: 54H15, 54E50, 28C10.

Key words and phrases: Polish group, non-locally compact, left Haar null set. 
The way this problem was originally stated by Solecki uses the notion of strong non-local compactness, introduced in [12]: a Polish group $G$ is said to be strongly non-locally compact if for every open neighborhood $U$ of the identity, there exists another neighborhood of the identity $V$ such that no finite family of two-sided translates of $V$ covers $U$.

Now, the question we are concerned with in this paper is whether nonlocal compactness implies strong non-local compactness (it is easy to see that the converse is true.) As in the compact case, this problem becomes trivial under an additional assumption that there exists a two-sided invariant compatible metric on $G$. Then one can show that the absence of strong non-local compactness implies that, for some neighborhood $U$ of the identity, every infinite sequence of elements of $U$ contains a convergent subsequence. The proof rests on the observation that the diameters of all two-sided translates of any given set are equal, so a straightforward diagonalization argument can be applied to find such a subsequence. It is known though that there exist Polish groups which do not admit a two-sided invariant compatible metric (see [4]), thus the approach sketched above is of no use in the general case.

Our first main result is a characterization of closed strongly non-locally compact subgroups of $S_{\infty}$, the group of all permutations of the natural numbers, in terms of group actions. A seemingly much stronger condition, found by Solecki, turns out to be equivalent to strong non-local compactness for closed subgroups of $S_{\infty}$.

Next, we prove that all members of a class of closed subgroups of $S_{\infty}$ including all oligomorphic groups are strongly non-locally compact. We also show that closed non-locally compact subgroups of products of locally compact groups are strongly non-locally compact. This is the only natural class of Polish strongly non-locally compact groups which is known to be closed under taking (closed non-locally compact) subgroups.

In Section 3, we briefly discuss connections of the concept of strong non-local compactness with left Haar null sets, and apply relevant results of Solecki to the classes of strongly non-locally compact groups investigated in Section 2 .

Most of the remaining part of the paper is devoted to the study of particular examples. We show that homeomorphisms of metrizable compactifications of $\mathbb{N}$ (investigated in [8] and [14]), the isometry group of the Urysohn space, and the unitary group of the separable Hilbert space are strongly nonlocally compact. Finally, we give a partial answer to the question whether Polish compact groups can be characterized in terms of actions, in a way analogous to Solecki's condition of strong non-local compactness.

All notions that are not explicitly defined are standard. Recall that a topological group is called Polish if its group topology is Polish, that is, 
separable and completely metrizable. A Polish group that we will be particularly interested in is $S_{\infty}$, the group of all permutations of the natural numbers with the pointwise convergence topology.

2. Strongly non-locally compact Polish groups. In [12], the following notion was introduced.

Definition. A Polish group is called strongly non-locally compact if for every neighborhood $V$ of the identity there exists a neighborhood $U$ of the identity such that $V$ cannot be covered by any finite family of two-sided translates of $U$.

Proposition 2 (Solecki, [12]). Suppose that $G$ is a Polish group such that for every neighborhood $U$ of the identity there is a continuous action of $G$ by uniformly continuous homeomorphisms on an infinite metric space $X$ and a natural number $m_{U}$ such that for any finite $F \subseteq X$ there are $F^{\prime} \subseteq X$ and $g_{n} \in U, n \in \mathbb{N}$, with

$$
\left|F^{\prime}\right| \leq m_{U}
$$

and $\left\{g_{n} F\right\}$ converging to $F^{\prime}$ in the sense of the Hausdorff metric. Then $G$ is strongly non-locally compact.

The next theorem states that, for closed subgroups of $S_{\infty}$, strong nonlocal compactness implies a strong version of the above condition.

THEOREM 3. Suppose that $G$ is a closed subgroup of $S_{\infty}$. If $G$ is strongly non-locally compact, then for every open neighborhood $U$ of the identity there exists a countable compact space $Z$, a point $z_{\infty} \in Z$, and a continuous action of $G$ on $Z$ such that for every finite $F \subseteq Z$ there exist $g_{n} \in U, n \in \mathbb{N}$, with

$$
g_{n} . F \rightarrow z_{\infty} .
$$

In the proof of Theorem 3, we will need to extend actions of open subgroups of $G$ to actions of $G$ in a suitable manner, and it turns out that the well-known canonical construction discovered by Mackey (see [7, p. 32]) will be useful for this purpose. For the reader's convenience, we briefly sketch this construction and its main properties.

Suppose that $H$ is a subgroup of an abstract group $G$, and $\alpha$ is an $H$-action on a set $X$. An action $\beta$ of $G$ on a set $Y$ can be constructed in such a way that $\alpha$ is the restriction of $\beta$ to the group $H$ and an $H$-invariant subset of $Y$. We will call this $\beta$ and $Y$ the Mackey extension of $\alpha$.

Consider $H$ as acting on $G$ by left multiplication, and let $Y=(X \times G) / H$ be the set of orbits of the action of $H$ on $X \times G$ by $h .(x, g)=(h . x, h g)$.

Now, $G$ acts on $Y$ by $\beta\left(g,\left[x, g^{\prime}\right]\right)=g \cdot\left[x, g^{\prime}\right]=\left[x, g^{\prime} g^{-1}\right]$. We check first that this is well-defined. If $\left[x, g^{\prime}\right]=\left[y, g^{\prime \prime}\right]$, then, for some $h \in H,\left(y, g^{\prime \prime}\right)=$ 
$h \cdot\left(x, g^{\prime}\right)$, so $h \cdot x=y, h g^{\prime}=g^{\prime \prime}$. Thus, $g \cdot\left[y, g^{\prime \prime}\right]=\left[y, g^{\prime \prime} g^{-1}\right]=\left[x, g^{\prime} g^{-1}\right]=$ $g \cdot\left[x, g^{\prime}\right]$.

Note that $f(x)=[x, 1]$ is injective: if $[x, 1]=[y, 1]$, then for some $h \in H$, $h . x=y, h 1=1$, so $h=1$, and $x=y$.

Since $[h . x, 1]=\left[x, h^{-1}\right]=h .[x, 1]$, we also have $f(h . x)=h . f(x)$ for $x \in X$. Thus, $f$ witnesses that $X$ can be viewed as a subset of $Y$ consisting of elements of the form $[x, 1]$, for $x \in X$. Under this identification, $\alpha$ is the restriction of $\beta$ to $X$.

The next proposition, even though stated in a much more general setting, will actually be applied only to actions of Polish groups on Polish (even discrete) spaces.

Proposition 4. Suppose that a topological group $H$ acts continuously on a Hausdorff space $X$ and $H$ is an open subgroup of a topological group $G$. Then the Mackey extension $(\beta, Y)$ of $\alpha$ can be made into a continuous action by choosing a topology on $Y$ that agrees with the topology on $X$.

Proof. We prove the proposition only for first countable $G$ and $X$. An inspection of the proof shows that the same argument goes through not only for sequences indexed by natural numbers, as considered in the proof, but also for generalized sequences (that is, Moore-Smith sequences, see [5, p. 52]).

Since $H$ is open in $G$, we can pick a discrete subset $C$ of $G$ such that any two distinct $c, c^{\prime} \in C$ belong to disjoint right cosets of $H$, and each right coset contains an element of $C$. Then the mapping $\pi: X \times C \rightarrow(X \times G) / G$ is easily seen to be bijective, so it gives rise to a topology on $Y=(X \times G) / G$ defined by

$$
B \subseteq Y \text { is open } \Leftrightarrow \pi^{-1}(B) \text { is open. }
$$

We show that the Mackey extension of $\alpha$ is continuous in this topology. Suppose that $\left[x_{n}, g_{n}\right] \rightarrow[x, g]$ and $f_{n} \rightarrow f$, where $f_{n}, g_{n}, f, g \in G, x_{n}, x \in X$. Observe that since $H$ is open and the group operations on $G$ are continuous, we can assume, without loss of generality, that $g_{n}=c, g=c$ for some $c \in C$, that is, $\left[x_{n}, c\right] \rightarrow[x, c]$. The very definition of the topology on $Y$ then gives $x_{n} \rightarrow x$.

Similarly, we can write $c f_{n}^{-1}=h_{n} c^{\prime}$ and $c f^{-1}=h c^{\prime}$ for some $h_{n}, h \in H$ and $c, c^{\prime} \in C$. Continuity of the group operations in $G$ guarantees that in this case $h_{n} \rightarrow h$. Since $H$ acts continuously on $X$, we have

$$
\begin{aligned}
f_{n} \cdot\left[x_{n}, c\right] & =\left[x_{n}, c f_{n}^{-1}\right]=\left[x_{n}, h_{n} c^{\prime}\right]=\left[h_{n}^{-1} \cdot x_{n}, c^{\prime}\right] \\
& \rightarrow\left[h^{-1} \cdot x, c^{\prime}\right]=\left[x, h c^{\prime}\right]=\left[x, c f^{-1}\right]=f \cdot[x, c],
\end{aligned}
$$

which finishes the proof.

The Mackey extension $(\beta, Y)$ of $\alpha$ with the topology defined in the proof of Proposition 4 will be called the continuous Mackey extension of $\alpha$. 
Now we are ready to prove Theorem 3 .

Proof of Theorem 3. As $G$ is a subgroup of $S_{\infty}$, there exists a neighborhood basis $\left\{H_{k}\right\}$ at the identity consisting of pointwise stabilizers of the sets $\{1, \ldots, k\} \subseteq \mathbb{N}$. By strong non-local compactness of $G$, we can choose a strictly increasing sequence $\left\{n_{k}\right\}$ so that no $H_{n_{k}}$ can be covered by finitely many two-sided translates of $H_{n_{k}+1}$. In particular, the orbit of $n_{k}+1$ under the action of $H_{n_{k}}$ on $\mathbb{N}$ is infinite.

Fix an open subgroup $H=H_{n_{k}}$ of $G$, for some $k \in \mathbb{N}$, and let $X$ be the infinite orbit of $n_{k}+1$. Denote by $\alpha$ the action of $H$ on $X$, and let $(\beta, Y)$ be the continuous Mackey extension of $\alpha$.

Claim. Every orbit of the restriction of $\beta$ to $H$ is infinite.

Fix $[n, g] \in Y$, and consider $h_{1}, \ldots, h_{r} \in H$ and $g_{1}, \ldots, g_{s} \in G$ such that the $h_{i}$ are in distinct left cosets of the stabilizer $H^{\prime}$ of $n$ in $H$, and the $g_{j}$ are in distinct right cosets of $H$. For $n_{i}=h_{i}(n), i \leq r$, we show that there is $b \in H$ such that $b .[n, g] \neq\left[n_{i}, g_{j}\right]$ for all $i \leq r$ and $j \leq s$.

Note that, since $n$ and $n_{k}+1$ are in the same $H$-orbit, $H^{\prime}$ is conjugate to $H_{n_{k}+1}$ in $H$. Therefore, strong non-local compactness of $G$ gives

$$
H \nsubseteq \bigcup_{i \leq r, j \leq s} g^{-1} H^{\prime} h_{i}^{-1} g_{j} .
$$

Suppose that $b .[n, g]=\left[n, g b^{-1}\right]=\left[n_{i}, g_{j}\right]$ for some $i \leq r$ and $j \leq s$. Then $h g b^{-1}=g_{j}$ for some $h \in H$ such that $h(n)=n_{i}$, that is, $h=h_{i} h^{\prime}, h^{\prime} \in H^{\prime}$. It follows that $b^{-1}=g^{-1} h^{\prime-1} h_{i}^{-1} g_{j}$. But (1) shows that there exists $b \in H$ which cannot be written in this way, so $b .[n, g] \neq\left[n_{i}, g_{j}\right]$ for all $i \leq r$ and $j \leq s$. This finishes the proof of the claim.

Recall the well-known Neumann lemma (see [3, p. 10]) which says that for any group $H$, if some finite family of left cosets of subgroups of $H$ covers $H$, then one of the subgroups has finite index. Since all orbits in $Y$ under the restriction of $\beta$ to $H$ are infinite, that is, the stabilizer of every element of $Y$ has infinite index in $H$, we find that, for any finite $F, K \subseteq Y$, there is a single $h \in H$ such that

$$
\text { h.F } \cap K=\emptyset .
$$

Indeed, otherwise, for any $h \in H$, there is $f \in F$ with $h . f \in K$, that is, $H$ is covered by finitely many left translates of stabilizers (in $H$ ) of elements of $F$. By Neumann's lemma, one of these stabilizers has finite index in $H$, which we know not to be the case.

Now we extend $\beta$ to an action on the one-point compactification $Z=$ $Y \cup z_{\infty}$ of $Y$ by putting $g . z_{\infty}=z_{\infty}$ for $g \in G$. Then for any finite $F \subseteq Z$, there is a sequence $\left\{h_{n}\right\}$ of elements in $H$ so that $h_{n} . F \rightarrow z_{\infty}$. 
Corollary 5. A closed subgroup $G$ of $S_{\infty}$ is strongly non-locally compact if and only if for every open neighborhood $U$ of the identity there exists a countable compact space $Z$, a point $z_{\infty} \in Z$, and a continuous action of $G$ on $Z$ such that for every finite $F \subseteq Z$ there exist $g_{n} \in U, n \in \mathbb{N}$, with

$$
g_{n} . F \rightarrow z_{\infty} .
$$

In certain situations, we are able to show that non-locally compact groups are strongly non-locally compact. We turn to these cases now.

The following lemma is essentially contained in [9].

Lemma 6. Let $G$ be an abstract group, and let $A_{1}, \ldots, A_{n}$ and $F^{\prime}$ be subsets of $G$, with $F^{\prime}$ finite, such that

$$
F^{\prime}\left(A_{1} \cup \cdots \cup A_{n}\right)=G .
$$

Then, for some $i \leq n$ and finite $F^{\prime \prime} \subseteq G$, we have $F^{\prime \prime} A_{i} A_{i}^{-1}=G$ or $F^{\prime \prime} A_{i}=G$.

Proof. Let $n=2$ and assume that $F^{\prime} A_{1} A_{1}^{-1} \neq G$, that is, there exists $g \in G$ such that $g A_{1} \cap F^{\prime} A_{1}=\emptyset$. Then $A_{1} \subseteq g^{-1} F^{\prime} A_{2}$, and $\left(F^{\prime} \cup g^{-1} F^{\prime}\right) A_{2}$ $=G$.

The general case is obtained by straightforward induction on $n$.

Proposition 7. Assume that $G$ is a closed subgroup of $S_{\infty}$, and $H, H^{\prime}$ are pointwise stabilizers in $G$ of finite sets $A, B$ respectively, with $A \subseteq B$. Suppose that $H \subseteq F_{1} H^{\prime} F_{2}$ for some finite $F_{1}, F_{2} \subseteq G$. Then there exists a stabilizer $H^{\prime \prime}$ in $G$ of a finite set $B^{\prime} \supseteq B$ and $g \in G$ such that $g^{-1} H^{\prime \prime} g$ is a finite index subgroup of $H$.

Proof. By Lemma 6, we can assume that $H \subseteq F H^{\prime} g$ for some finite $F \subseteq G, g \in G$ : we take $F^{\prime}=\{1\}$, and $A_{i}$ to be sets of the form $f_{1} H^{\prime} f_{2} \cap H$, $f_{1} \in F_{1}, f_{2} \in F_{2}$.

Fix $f \in F$. Let $H^{\prime \prime}$ be the pointwise stabilizer of the set $B \cup g(A)$, and $h_{1}$ an element of $H^{\prime}$ such that there exists $f \in F$ such that $h_{1}(g(a))=f^{-1}(a)$ for every $a \in A$. Then, for any $h^{\prime} \in H^{\prime}$, we have $f h^{\prime} g \in H^{\prime}$ if and only if $h^{\prime} \in h_{1} H^{\prime \prime}$.

It follows that $H=F^{\prime} H^{\prime \prime} g$ for some finite $F^{\prime} \subseteq G$. In particular, $F^{\prime} g$ $\subseteq H$, that is, $F^{\prime} \subseteq H g^{-1}$, so there is $F^{\prime \prime} \subseteq H$ such that $H=F^{\prime \prime} g^{-1} H^{\prime \prime} g$, and $H^{\prime \prime} \leq H$.

COROLlary 8. Suppose that $G$ is a closed subgroup of $S_{\infty}$, and there exists in $G$ a neighborhood basis $\left\{H_{n}\right\}$ of the identity consisting of pointwise stabilizers of finite sets such that for each $n \in \mathbb{N}$, the natural action of $H_{n}$ on $\mathbb{N}$ has only finitely many finite orbits. Then $G$ is strongly non-locally compact. 
Proof. It suffices to show that for every $n \in \mathbb{N}$ there exists $m \in M$ such that $H_{n}$ cannot be covered by any finite union of two-sided translates of $H_{m}$. Fix $n \in \mathbb{N}$, let $K$ be the size of the set of all elements in finite orbits of the action of $H_{n}$, and let $H^{\prime}$ be the pointwise stabilizer in $G$ of the set $B=\{1, \ldots, K+1\}$. If $H_{n}$ can be covered by finitely many two-sided translates of $H^{\prime}$, then, by the previous proposition, there exists a pointwise stabilizer $H^{\prime \prime} \leq H^{\prime}$ of a set $B^{\prime} \supseteq B$, and $g \in G$ such that $g^{-1} H^{\prime \prime} g$ has finite index in $H_{n}$. But $g^{-1} H^{\prime \prime} g$ is a pointwise stabilizer of $g^{-1}\left(B^{\prime}\right)$ which has size at least $K+1$, so some $b \in B^{\prime \prime}$ is in an infinite orbit of $H_{n}$. This clearly implies that $g^{-1} H^{\prime \prime} g$ has infinite index in $H$, which leads to a contradiction. Thus, the group $H_{n}$ cannot be covered by finitely many two-sided translates of $H^{\prime}$ or any $H_{m}$ with $H_{m} \leq H^{\prime}$.

Recall that a subgroup $G$ of $S_{\infty}$ is called oligomorphic if for each natural number $k$, the natural action of $G$ on $\mathbb{N}^{k}$ has only finitely many orbits (see [3] for more on this important notion). It is straightforward to check that oligomorphic groups satisfy the assumptions of the above corollary. Thus, we have

COROllary 9. Oligomorphic groups are strongly non-locally compact.

It turns out that all closed non-locally compact subgroups of products of locally compact Polish groups are strongly non-locally compact. This is the only situation where strong non-local compactness is known to be closed under taking (closed non-locally compact) subgroups.

Theorem 10. Let $\left\{G_{n}\right\}$ be a countable family of locally compact Polish groups and let $G=\prod G_{n}$. Every non-locally compact closed subgroup $H$ of $G$ is strongly non-locally compact.

Proof. Let $H$ be a non-locally compact closed subgroup of $G$, and let $U$ be a fixed open neighborhood of the identity in $H$. Since the closure of $U$ is not compact, there must exist $n_{U} \in \mathbb{N}$ such that the closure of the projection $\pi_{n_{U}}(U)$ of $U$ on the $n_{U}$ th coordinate is non-compact. Fix a sequence $\left\{h_{k}\right\}$ in $U$ such that no infinite subset of $\left\{\pi_{n_{U}}\left(h_{k}\right)\right\}$ has compact closure in $G_{n_{U}}$, and consider an action $\alpha_{U}$ of $H$ on $G_{n_{U}}$ by left translations:

$$
h . g=\pi_{n_{0}}(h) g \quad \text { for } h \in H, g \in G_{n_{U}} .
$$

Clearly, no subsequence of any sequence of the form $\left\{\pi_{n_{U}}\left(h_{k}\right) g\right\}, g \in G_{n_{U}}$, has compact closure. Observe that this implies that, for any finite $F \subseteq$ $G_{n_{U}}$ and compact $K \subseteq G_{n_{U}}$, there exists $h_{k}$ such that $\pi_{n_{0}}\left(h_{k}\right) F \cap K=\emptyset$. Otherwise, for some compact $K$ there is $g \in G_{n_{U}}$ such that $\pi_{n_{U}}\left(h_{k}\right) g \in K$ for infinitely many $k \in \mathbb{N}$, which contradicts our choice of $\left\{h_{k}\right\}$. The extensions of the actions $\alpha_{U}$ of $H$ on $G_{n_{U}}$ to the actions on one-point compactifications of $G_{n_{U}}$ by fixing the point at infinity witness that $H$ satisfies the assumptions of Proposition 2. Therefore, it is strongly non-locally compact. 
We finish this section with a couple of additional remarks.

From Lemma 6 one can derive several interesting observations. First of all, it implies that if $H$ is a normal subgroup of $G$ that can be covered by finitely many two-sided translates of some subgroup $H^{\prime}$, then it can also be covered by finitely many left translates of $H^{\prime}$. This is because right cosets of $H$ are also left cosets, so, for $F$ and $g$ such that $H \subseteq F H^{\prime} g$, we have $g H=H g^{-1} \subseteq F H^{\prime}$, that is, $H \subseteq g^{-1} F H^{\prime}$. This implies that a non-locally compact group with a basis of neighborhoods of the identity consisting of normal subgroups is strongly non-locally compact. Yet, since it is well known that this implies that such groups admit a two-sided invariant metric, the above observation does not yield any new conditions implying strong nonlocal compactness.

Notice also that if $G$ contains an open subgroup $H$ of finite index in $G$ that can be covered by finitely many two-sided translates of any neighborhood of the identity, then $G$ is compact. This generalizes Lemma 1.1 from [11].

Fix a left invariant metric $d$ on $G$ and a sequence of neighborhoods $U_{n}$ of the identity with the diameter of $U_{n} U_{n}^{-1}$ converging to 0 , and let $F_{n} \subseteq G$ and $g_{n} \in G$ be such that $H \subseteq F_{n} U_{n} U_{n}^{-1} g_{n}$. Existence of such $F_{n}, g_{n}$ is warranted by Lemma 6 . Then we can pick infinitely many $n_{k} \in \mathbb{N}$ such that $H g_{n_{k}}^{-1}=H g^{\prime-1}$ for some $g^{\prime} \in G$. By continuity of group operations, if $\operatorname{diam}\left(U_{n} U_{n}^{-1}\right) \rightarrow 0$, then $\operatorname{diam}\left(U_{n} U_{n}^{-1} g^{\prime}\right) \rightarrow 0$, so $H$ can be covered by finite families of arbitrarily small neighborhoods of the identity in the sense of $d$.

Now we can repeat the same argument for the right invariant metric $d^{\prime}(g, h)=d\left(g^{-1}, h^{-1}\right), g, h \in G$. Therefore $H$ can be covered by finite families of arbitrarily small neighborhoods of the identity in the sense of $d+d^{\prime}$, which is complete. Compactness of $H$ follows by a standard diagonalization argument.

Finally, we observe that preimages of strongly non-locally compact groups are strongly-non locally compact.

Proposition 11. Assume that $\phi: H \rightarrow G$ is a continuous surjective homomorphism of Polish groups. Then strong non-local compactness of $G$ entails strong non-local compactness of $H$.

Proof. We show the contrapositive. Assume that $H$ is not strongly nonlocally compact, and let $V^{\prime}$ be a neighborhood of the identity in $H$ that can be covered by a finite number of two-sided translates of any neighborhood of the identity. Since $\phi$ is open (see [7]), $V=\phi\left(V^{\prime}\right)$ is a neighborhood of the identity in $G$. Fix a neighborhood $U$ of the identity in $G$. Find an open $U^{\prime}$ in $H$ such that $\phi\left(U^{\prime}\right) \subseteq U$, and let $F_{1}, F_{2} \subseteq H$ be finite sets such that $F_{1} U^{\prime} F_{2} \supseteq V^{\prime}$. Clearly, $V \subseteq \phi\left(F_{1}\right) U \phi\left(F_{2}\right)$, so $G$ is not strongly non-locally compact either. 
3. Left Haar null sets. Results from the previous section have applications to the theory of Haar null sets in non-locally compact Polish groups. In [12], Solecki introduced the following definition which is a natural generalization of the standard notion of Haar null subsets of a locally compact group $G$, that is, subsets of measure zero with respect to the Haar measure on $G$ :

Definition. A subset $A$ of a Polish group $G$ is called left Haar null if there is a universally measurable set $B \supseteq A$ and a Borel probability measure $\mu$ on $G$ such that $\mu(g B)=0$ for all $g \in G$.

One can also define Haar null sets by requiring that $\mu(f B g)=0$ for all $f, g \in G$ in the above definition. Since Haar null sets are left Haar null, Theorem 14 holds for them as well.

The behavior of left Haar null sets depends on the structure of $G$. Solecki proved that if $G$ is amenable at 1 (see [12, p. 3] for definition), then the family of left Haar null sets forms a proper $\sigma$-ideal, and it has the Steinhaus property, that is, if $A \subseteq G$ is universally measurable and not left Haar null, then $1 \in \operatorname{int}\left(A^{-1} A\right)$. On the other hand, strong non-local compactness implies that Haar null sets are not ccc ([13]), and if we additionally assume that $G$ contains a non-discrete free group all of whose subgroups are discrete (a free subgroup at 1), then they do not have the Steinhaus property either $([12])$.

The assumption of strong non-local compactness in these results shows up only in the following lemma ([12, Lemma 4.4]). We denote by $\mathcal{K}(X)$ the space of all compact subsets of $X$ with the Vietoris topology. (See [6].)

Lemma 12 (Solecki, [12]). Let $G$ be a strongly non-locally compact Polish group. Let $X$ be a Borel subset of a Polish space and let $F: X \rightarrow \mathcal{K}(G)$ be Borel. Then there exists a Borel function $X \ni x \mapsto g_{x} \in G$ such that for any distinct $x_{1}, x_{2} \in X$,

$$
g_{x_{1}} F\left(x_{1}\right) \cap g_{x_{2}} F\left(x_{2}\right)=\emptyset .
$$

COROLlary 13 (Solecki, [12]). Let $G$ be a strongly non-locally compact Polish group. Then the family of all left Haar null sets is not ccc.

Proof. Take $X=2^{\mathbb{N}} \times \mathcal{K}(G)$ and let $F$ be the projection of $X$ onto $\mathcal{K}(G)$. Apply the lemma above to get $g_{(K, z)}$. Define now

$$
A_{z}=\bigcup\left\{g_{(K, z)} F(K): K \in \mathcal{K}(G)\right\} .
$$

It is easy to check that all $A_{z}$ are analytic, so universally measurable, and $A_{z_{1}} \cap A_{z_{2}}=\emptyset$ for distinct $z_{1}, z_{2}$. Now, since for every Borel probability measure on a non-locally compact space there exists a compact $K$ of positive measure and $K \subset g_{(K, z)}^{-1} A_{z}$, none of the $A_{z}$ can be left Haar null. 
Combining results from Section 3 with the above considerations, we get:

Theorem 14. Let $G$ be a Polish group that is either

(a) a closed subgroup of $S_{\infty}$ with a neighborhood basis at the identity consisting of open subgroups whose actions on $\mathbb{N}$ have only finitely many finite orbits; or

(b) a closed non-locally compact subgroup of $\prod G_{n}$, where each $G_{n}$ is a locally compact Polish group.

Then

(i) the family of left Haar null subsets of $G$ is not ccc;

(ii) if $G$ has a free subgroup at 1 , then $G$ does not have the Steinhaus property.

4. Examples. In this section, we consider several examples of strongly non-locally compact groups that are not covered by results in Section 2.2.

Usually Polish groups come equipped with natural actions: groups of homeomorphisms of compact spaces, groups of isometries of Polish spaces, automorphism groups of countable models and so forth. The groups studied in Examples 2-4 below are interesting in this respect because their natural actions do not fit the assumption of Proposition 2. Example 2 is a group of homeomorphisms of the interval $[0,1]$ with a metric defined in a way that mappings "squeezing" points cannot be too close to the identity. Examples 3 and 4 are groups of isometries of Polish spaces, so obviously there is no possibility for the actions of these groups on the spaces involved to change distances between points.

Example 1 is actually a class of groups that do not have in general a neighborhood basis at the identity consisting of subgroups, but an argument similar to the one used in the proof of Theorem 3 allows us to show that they are strongly non-locally compact.

Example 1. Consider a metrizable compactification $\widetilde{X}$ of the natural numbers $\mathbb{N}$, that is, a metrizable compact space $\widetilde{X}$ containing a countable dense discrete subset. The homeomorphism group $\operatorname{Hom}(\widetilde{X})$ of $\widetilde{X}$, equipped with the uniform convergence topology, is a Polish group. These groups were studied in [8] and [14]. In particular, Tsankov proved that every Polish group is a continuous homomorphic image of a group of the form $\operatorname{Hom}(\widetilde{X})$, $\widetilde{X}$ a metrizable compactification of $\mathbb{N}$, which means that the class of such homeomorphism groups is large.

Proposition 15. For every metrizable compactification $\widetilde{X}$ of $\mathbb{N}$, that is, a compact metric space $\widetilde{X}$ containing a countable dense discrete subset, the group $\operatorname{Hom}(\widetilde{X})$ is strongly non-locally compact. 
Proof. $\mathbb{N}$ as a subset of $\tilde{X}$ is a metric space with a totally bounded metric $\varrho$ inherited from $\widetilde{X}$. Note that $G=\operatorname{Hom}(\widetilde{X})$ can be naturally identified with the group of all uniformly continuous homeomorphisms of $(\mathbb{N}, \varrho)$ with the uniform convergence topology.

We show that the continuous extension of the natural action of $G$ on $\mathbb{N}$ to an action on the one-point compactification $\mathbb{N}_{\infty}$ of $\mathbb{N}$, where we put $g \cdot n_{\infty}=n_{\infty}$ for every $g \in G$, witnesses that the assumptions of Proposition 2 are met for $G$.

Denote by $U_{\varepsilon}$ the open ball around the identity in $G$ of radius $\varepsilon>0$ in the supremum metric on $G$ and fix a dense subset $\left\{q_{i}\right\}$ in $\widetilde{X} \backslash \mathbb{N}$. For a fixed $\varepsilon>0$, let $\left\{A_{i}\right\}$ be a family of subsets of $\mathbb{N}$ such that:

(i) the $A_{i}$ are pairwise disjoint;

(ii) elements of $A_{i}$ converge to $q_{i}$;

(iii) the distance between any $a \in A_{i}$ and $q_{i}$ is less than $\varepsilon / 2$;

(iv) every $a \in \mathbb{N}$ whose distance from $\widetilde{X} \backslash \mathbb{N}$ is less than $\varepsilon / 2$ is in some $A_{i}$.

Let $H$ be the group of those $g \in G$ that have the following properties:

(a) $g\left(A_{i}\right)=A_{i}$;

(b) $g$ fixes all elements outside of $\bigcup_{i} A_{i}$;

(c) $g$ restricted to $A_{i}$ moves only finitely many elements in every $A_{i}$.

Clearly $H \subseteq U_{\varepsilon}$. Since $\varrho$ is totally bounded, $\widetilde{X} \backslash \bigcup A_{i}$ is finite, say of size $k \in \mathbb{N}$. Consider a finite $F \subseteq \mathbb{N}_{\infty}$. Since the infinite sets $A_{i}$ are orbits of $H$, for any given $n \in \mathbb{N}$ using Neumann's lemma we can find $h_{n} \in H$ such that $h_{n} . f>n$ for all $f \in F$ except for those that are not in $\bigcup A_{i}$. But there cannot be more than $k$ such elements so $h_{n} . F$ converges to a set of size at most $k+1$. This proves the proposition.

It is obvious that every Polish group $G$ is a continuous homomorphic image of a strongly non-locally compact group. For instance, for a given $G$, take $G \times S_{\infty}$ and the projection of this group onto $G$. However, the results from [14] combined with Proposition 15 give a little more. Recall that a topological space $X$ is called almost zero-dimensional if for every $x \in X$ there exists a neighborhood basis at $x$ consisting of sets that are intersections of clopen sets.

Corollary 16. Every Polish group is a continuous homomorphic image of an almost zero-dimensional strongly non-locally compact group.

Proof. By results from [14], every homeomorphism group $\operatorname{Hom}(\widetilde{X})$ of a metrizable compactification $\widetilde{X}$ of $\mathbb{N}$ is almost zero-dimensional, and every Polish group is a continuous image of such a $\operatorname{Hom}(\widetilde{X})$. Together with Proposition 15 , this implies the statement of the corollary. 
Example 2. Let $G$ be the group of all increasing homeomorphisms of $[0,1]$ such that, for every $g \in G$, the derivatives $g^{\prime}$ and $\left(g^{-1}\right)^{\prime}$ exist everywhere and are continuous. Define the following metric $\varrho$ on $G$ :

$$
\varrho(f, g)=\sup _{x}\left|f^{\prime}(x)-g^{\prime}(x)\right|+\sup _{x}\left|\left(f^{-1}\right)^{\prime}(x)-\left(g^{-1}\right)^{\prime}(x)\right|, \quad f, g \in G .
$$

Proposition 17. The group $G$ with the topology induced by $\varrho$ is a Polish strongly non-locally compact group.

We will need two standard facts about $C([0,1])$, the space of all continuous real functions on $[0,1]$ with the uniform convergence topology (see [10]).

Lemma 18. $C([0,1])$ is Polish and the supremum metric is complete on $C([0,1])$.

Lemma 19. Suppose that for some $f_{n}, f, g \in C([0,1]), n \in \mathbb{N}$, all derivatives $f_{n}^{\prime}$ exist everywhere and are continuous. If $f_{n} \rightarrow f$ and $f_{n}^{\prime} \rightarrow g$ uniformly, then $f^{\prime}$ exists everywhere and is equal to $\mathrm{g}$.

Proof of Proposition 17. First, observe that if $f_{n} \rightarrow f$ in $\varrho$, then $f_{n}^{\prime} \rightarrow g$ and $\left(f_{n}^{-1}\right)^{\prime} \rightarrow g_{1}$ uniformly for some $g, g_{1} \in C([0,1])$. Also, for $f(x)=$ $\int_{0}^{x} g(t) d t$ and $f_{1}(x)=\int_{0}^{x} g_{1}(t) d t$ we have $f_{n} \rightarrow f$ and $f_{n}^{-1} \rightarrow f_{1}$ uniformly. By Lemma $19, f^{\prime}=g, f_{1}^{\prime}=g_{1}$ and $f=f_{1}^{-1}$, that is, $f \in G$.

Completeness and separability of $\varrho$ follow from Lemma 18. Therefore, $\varrho$ induces a Polish topology on $G$.

Denote by $U_{\varepsilon}$ the open ball around the identity with radius $\varepsilon>0$, and fix $f_{1}, \ldots, f_{n}, h_{1}, \ldots, h_{n}$ in $G$. We construct an element $g \in U_{\varepsilon}$ such that $f_{i} g h_{i} \notin U_{\varepsilon / 2}$ for every $i \leq n$, which is equivalent to showing that the two-sided translates $f_{i}^{-1} U_{\varepsilon / 2} h_{i}^{-1}, i \leq n$, do not cover $U_{\varepsilon}$.

The construction is as follows. Fix pairwise distinct $x_{1}, \ldots, x_{n} \in[0,1]$ such that

$$
0<h_{i}\left(x_{i}\right)<h_{j}\left(x_{j}\right)<1 \quad \text { for } i<j .
$$

This can be done easily because the $h_{i}$ are bijective. Now let

$$
M_{i}=f_{i}^{\prime}\left(h_{i}\left(x_{i}\right)\right) h_{i}^{\prime}\left(x_{i}\right), \quad m_{i}= \begin{cases}1 & \text { if } M_{i} \geq 1, \\ -1 & \text { if } 0 \leq M_{i}<1 .\end{cases}
$$

We construct $g^{\prime}$ in the following way. For each $i \leq n$ find $z_{1}^{i}, \ldots, z_{7}^{i} \in[0,1]$ such that:

- $h_{i-1}\left(x_{i-1}\right)<z_{j}^{i}<z_{j+1}^{i}<h_{i+1}\left(x_{i+1}\right)$;

- $z_{4}^{i}=h_{i}\left(x_{i}\right)$;

- $z_{1}^{i}, \ldots, z_{7}^{i}$ are equally spaced.

Put

$$
\begin{aligned}
& g^{\prime}\left(z_{1}^{i}\right)=g^{\prime}\left(z_{3}^{i}\right)=g^{\prime}\left(z_{5}^{i}\right)=g^{\prime}\left(z_{7}^{i}\right)=1, \\
& g^{\prime}\left(z_{2}^{i}\right)=g^{\prime}\left(z_{6}^{i}\right)=1-m_{i} \varepsilon / 4, \quad g^{\prime}\left(z_{4}^{i}\right)=1+m_{i} \varepsilon / 2 .
\end{aligned}
$$


Let $g^{\prime}$ be the simplest piecewise linear function interpolating these values. Thus, $g^{\prime}$ is most of the time close to 1 and its values at $z_{j}^{i}$ force it to look partially like uneven saw-teeth. It is easy to check that

$$
\int_{0}^{h_{i}\left(x_{i}\right)} g^{\prime}(x) d x=h_{i}\left(x_{i}\right),
$$

that is, $g\left(h_{i}\left(x_{i}\right)\right)=h_{i}\left(x_{i}\right)$ and $g \in G$.

Now consider the function $f_{i} \circ g \circ h_{i}$. Its derivative is given by the formula

$$
\left(f_{i} \circ g \circ h_{i}\right)^{\prime}(x)=f_{i}^{\prime}\left(g\left(h_{i}(x)\right)\right) g^{\prime}\left(h_{i}(x)\right) h_{i}^{\prime}(x),
$$

so

$$
\left(f_{i} \circ g \circ h_{i}\right)^{\prime}\left(x_{i}\right)=f_{i}^{\prime}\left(h_{i}\left(x_{i}\right)\right) h_{i}^{\prime}(x) g^{\prime}\left(h_{i}\left(x_{i}\right)\right)=M_{i} g^{\prime}\left(h_{i}\left(x_{i}\right)\right)
$$

(because $\left.g\left(h_{i}\left(x_{i}\right)\right)=h_{i}\left(x_{i}\right)\right)$. If $M_{i} \geq 1$, then it is larger than $1+\varepsilon / 2$, otherwise it is smaller than $1-\varepsilon / 2$, so in any case $x_{i}$ witnesses the inequality $\varrho\left(f_{i} \circ g \circ h_{i}, \mathrm{Id}\right)>\varepsilon / 2$ for $i \leq n$.

Example 3. In the next example, we analyse the group of all isometries of the Urysohn space $\mathbb{U}$, considered with the pointwise convergence topology. For our present purposes, it suffices to know that $\mathbb{U}$ is the unique (up to isometry) Polish metric space, which is

(a) universal, that is, $\mathbb{U}$ contains an isometric copy of every Polish space;

(b) ultrahomogeneous, that is, for every finite metric space $B \supseteq A$, every isometric embedding $\phi: A \rightarrow \mathbb{U}$ can be extended to an isometric embedding $\psi: B \rightarrow \mathbb{U}$. In particular, every isometry $\phi: A \rightarrow B$, where $A, B$ are finite subsets of $\mathbb{U}$, can be extended to an isometry of $\mathbb{U}$.

Proposition 20. The group Iso(U) of all isometries of the Urysohn space is strongly non-locally compact.

Proof. Fix $x_{1}, \ldots, x_{n} \in \mathbb{U}, \varepsilon>0$, and consider the neighborhood of the identity

$$
U_{x_{1}, \ldots, x_{n}, \varepsilon}=\left\{h \in \operatorname{Iso}(U): d\left(h\left(x_{i}\right), x_{i}\right)<\varepsilon\right\} .
$$

We show that for any $\delta<\varepsilon$ no finite family of two-sided translates of $V=U_{x_{1}, \ldots, x_{n}, \delta}$ covers $U_{x_{1}, \ldots, x_{n}, \varepsilon}$.

Fix finite $F, G \subseteq \operatorname{Iso}(\mathbb{U})$, and let $a_{f}^{1}, \ldots, a_{f}^{n}, b_{g}^{1}, \ldots, b_{g}^{n}, f \in F, g \in G$, be defined by

$$
a_{f}^{i}=f\left(x_{i}\right), \quad b_{g}^{i}=g^{-1}\left(x_{i}\right),
$$

so that

$$
f V g=\bigcup_{f \in F, g \in G}\left\{h \in \operatorname{Iso}(\mathbb{U}): d\left(h\left(a_{f}^{i},\right), b_{g}^{i}\right)<\delta \text { for } i \leq n\right\} .
$$


Fix $\varepsilon_{0}$ with $\delta<\varepsilon_{0}<\varepsilon$, and let

$$
A=\left\{a_{f}^{i}, b_{g}^{i}: f \in F, g \in G, i \leq n\right\} \cup\left\{x_{1}, \ldots, x_{n}\right\} .
$$

Let $A^{\prime}$ be a disjoint copy of $A$, and define $B=A \cup A^{\prime}$, endowed with the following metric $d_{B}$ :

$$
d_{B}(a, b)= \begin{cases}d(a, b) & \text { if } a, b \in A \text { or } a, b \in A^{\prime}, \\ d\left(a, b^{\prime}\right)+\varepsilon_{0} & \text { if } a \in A \text { and } b \in A^{\prime}, \text { where } b^{\prime} \text { is the copy of } b .\end{cases}
$$

It is easy to see that $d_{B}$ is a metric indeed, and that every element of $A^{\prime}$ is at least $\varepsilon_{0}$ away from any element of $A^{\prime}$. By ultrahomogeneity of $\mathbb{U}$, we may assume that $B$ is a metric subspace of $\mathbb{U}$, that is, $A^{\prime}$ is also a subset of $\mathbb{U}$, and $d_{B}$ is the restriction of $d$ to $B$.

Now, since $A$ and $A^{\prime}$ are isometric and $\mathbb{U}$ is ultrahomogeneous, there is $h \in \operatorname{Iso}(\mathbb{U})$ that maps every element of $A$ to its copy in $A^{\prime}$. Observe that $h \in U_{x_{1}, \ldots, x_{n}, \varepsilon}$ because $d\left(x_{i}, h\left(x_{i}\right)\right)=\varepsilon_{0}<\varepsilon$ for $i \leq n$. On the other hand, such an $h$ clearly cannot be in $F V G$, because $h\left(a_{f}^{i}\right) \in A^{\prime}$ for every $f \in F$ and $i \leq n$, so its distance from any $b_{g}^{i}$ must be greater than $\varepsilon_{0}>\delta$. This finishes the proof, since $U_{x_{1}, \ldots, x_{n}, \varepsilon}$ was arbitrary.

REMARK. The same argument proves strong non-local compactness for Iso $\left(\mathbb{U}_{1}\right)$, where $\mathbb{U}_{1}$ is the Urysohn space with metric bounded by 1 .

Example 4. As the following argument shows, the group of all unitary operators of the separable Hilbert space with pointwise convergence topology is strongly non-locally compact.

Proposition 21. The group $U(H)$ of unitary operators on the infinitedimensional separable Hilbert space is strongly non-locally compact.

Proof. Fix an orthonormal base $\left\{e_{i}\right\}$ of $H$, and note that in the pointwise convergence topology on $U(H)$ the sets

$$
U_{n, \varepsilon}=\left\{f \in U(H):\left\|f\left(e_{i}\right)-e_{i}\right\|<\varepsilon, i \leq n\right\}
$$

form a basis of neighborhoods of the identity. We need the following fact:

Lemma 22. For every $n \in \mathbb{N}$ there exists $\delta>0$ such that for any $f, g \in$ $U(H)$ there are $x, y \in H$ with

$$
\|h(x)-y\|<\delta, \quad\left\|x-e_{j}\right\|>\delta \quad \text { for all } j \leq n \text { and } h \in f U_{n+1, \delta} g .
$$

The proof is straightforward.

Fix $\varepsilon>0, n \in \mathbb{N}$ and let $\delta>0$ be as in the lemma. Also, for given $f_{1}, \ldots, f_{k}, g_{1}, \ldots, g_{k}$ in $U(H)$ let $x_{i}, y_{i}$ be as in the lemma for each $f_{i}, g_{i}$. We can write each $x_{i}$ as

$$
x_{i}=x_{i}^{\prime}+x_{i}^{\prime \prime},
$$

where $\left\|x_{i}^{\prime}\right\|>\delta$ and $x_{i}^{\prime} \perp x_{i}^{\prime \prime}, x_{i}^{\prime} \perp e_{j}$ for $j \leq n$. 
Let

$$
X=\operatorname{span}\left\{x_{1}^{\prime}, \ldots, x_{k}^{\prime}\right\}, \quad Y=\operatorname{span}\left\{y_{1}, \ldots, y_{k}\right\}, \quad E=\operatorname{span}\left\{e_{1}, \ldots, e_{n}\right\},
$$

and fix a linear subspace $Z$ of $H$, orthogonal to $Y$ and $E$, with $\operatorname{dim} Z=$ $\operatorname{dim} X$. There exists $g \in U(H)$ such that $g\left(e_{j}\right)=e_{j}$ for $j \leq n$, and $g\left(x_{i}\right) \in Z$ for $i \leq k$. Since

$$
\left\|g\left(x_{i}\right)-y_{i}\right\|^{2}=\left\|g\left(x_{i}^{\prime}\right)+g\left(x_{i}^{\prime \prime}\right)-y\right\|^{2}=\left\|g\left(x_{i}^{\prime}\right)\right\|^{2}+\left\|g\left(x_{i}^{\prime \prime}\right)-y\right\|^{2}>\delta^{2},
$$

we have $g \notin f_{i} U_{n+1, \delta} g_{i}$ for every $i \leq k$.

REMARK. Notice that the proof of the above proposition also shows that the sets

$$
U_{n}=\left\{g \in U(H): g\left(e_{i}\right)=e_{i} \text { for } i \leq n\right\}, \quad n \in \mathbb{N},
$$

cannot be covered by finitely many two-sided translates of sufficiently small neighborhoods of the identity. This is interesting because each $U_{n}$ has empty interior.

5. Actions of non-compact Polish groups. In this section, we give a partial positive answer to the following question, motivated by Proposition 2 . Is it true that for every non-compact Polish group $G$ there exists an action of $G$ by uniformly continuous homeomorphisms on an infinite metric space $X$ and a natural number $N$ such that for any finite $F \subseteq X$ there is some $F_{0} \subseteq X$ and a sequence $\left\{g_{n}\right\}$ in $G$ with

$$
\left|F_{0}\right| \leq N \quad \text { and } \quad g_{n} F \rightarrow F_{0} \quad \text { as } n \rightarrow \infty ?
$$

Theorem 23. Assume that a Polish group $G$ admits a left invariant unbounded metric. Then there exists a continuous action of $G$ on an infinite compact Polish space $X$, a point $x_{\infty} \in X$, and a sequence $\left\{g_{n}\right\}$ in $G$ such that

$$
g_{n} F \rightarrow x_{\infty} \quad \text { as } n \rightarrow \infty
$$

for any finite $F \subseteq X$.

Definition. Let $(Y, d)$ be a metric space. We define

$$
C L(Y)=\{d(A, \cdot): Y \rightarrow \mathbb{R}: A \text { is a closed non-empty subset of } Y\},
$$

where $d(A, \cdot)$ assigns to every $y \in Y$ its distance from $A$.

We view $C L(Y)$ as a subset of the space $C_{\mathrm{p}}(Y, \mathbb{R})$ of all continuous real functions on $Y$ with the pointwise convergence topology. Now, we have

Proposition 24 (Beer, [2]). Let $Y$ be a Polish space with a compatible metric $d$ on it. Then, for every $y_{0} \in Y, \overline{C L(Y)} \subseteq C_{\mathrm{p}}(Y, \mathbb{R})$ is the union of the family $\left\{\Delta_{n}^{Y}\right\}$ of sets defined by $\Delta_{n}^{Y}=\left\{f \in \overline{C L(Y)}: f\left(y_{0}\right) \leq n\right\}$, and for every $n \in \mathbb{N}$, 
(a) $\Delta_{n}^{Y}$ is compact;

(b) $\Delta_{n}^{Y} \subseteq \operatorname{Int}\left(\Delta_{n+1}^{Y}\right)$.

In particular, $\overline{C L(Y)}$ is Polish.

Proof of Theorem 23. Let $d$ be an unbounded left invariant metric on $G$. Let $x_{g}, g \in G$, stand for the distance function $d(g, \cdot): G \rightarrow \mathbb{R}$. Note that $\overline{X^{\prime}}$, for $X^{\prime}=\left\{x_{g}: g \in G\right\}$, is a closed subset of $C L(G)$, so it is Polish locally compact by Proposition 24. It is not compact because of unboundedness of $d$.

Consider the action $\alpha$ of $G$ on $\overline{X^{\prime}}$ given by

$$
g . f(a)=f\left(g^{-1} a\right), \quad f \in \overline{X^{\prime}} .
$$

To make sure that this is an action, two observations suffice. The first is that, after identifying $g \in G$ with $x_{g} \in X^{\prime}, \alpha$ is an action of $G$ on itself by left translations, so $X^{\prime}$ is an orbit under $\alpha$. The second observation is that $g . f \in \overline{X^{\prime}}$ for any $f \in \overline{X^{\prime}}$ and $g \in G$. This is because if $x_{h_{n}} \rightarrow f \in \overline{X^{\prime}}$ pointwise, then

$$
x_{g h_{n}}(a)=d\left(g h_{n}, a\right)=d\left(h_{n}, g^{-1} a\right)=x_{h_{n}}\left(g^{-1} a\right) \rightarrow f\left(g^{-1} a\right)=g \cdot f(a)
$$

for every $a \in G$. Therefore, $x_{g h_{n}} \rightarrow g$. $f$ pointwise, and since $f$ was arbitrary, $\alpha$ is well-defined on $\overline{X^{\prime}}$.

By Proposition 24 and Theorem 3.4 from [1], continuity of $\alpha$ follows from continuity in each coordinate, and this is straightforward to check: assume that $g_{n} \rightarrow g$ in $G$ and $f_{n} \rightarrow f$ in $\overline{X^{\prime}}$ pointwise. Then for every $a \in G$,

$$
\begin{gathered}
g_{n} \cdot f(a)=f\left(g_{n}^{-1} a\right) \rightarrow f\left(g^{-1} a\right)=g \cdot f(a), \\
\left(g \cdot f_{n}\right)(a)=f_{n}\left(g^{-1} a\right) \rightarrow f\left(g^{-1} a\right)=(g \cdot f)(a) .
\end{gathered}
$$

Let $\Delta_{n}^{G}$ be as in Proposition 24 for $y_{0}=1$, and put $\Delta_{n}=\Delta_{n}^{G} \cap \overline{X^{\prime}}$. We show that for every $n \in \mathbb{N}$ there is $g_{n} \in G$ such that $g_{n} . \Delta_{n} \cap \Delta_{n}=\emptyset$. As every finite $F \subseteq \overline{X^{\prime}}$ is contained in some $\Delta_{n_{0}}$, this implies that the extension of $\alpha$ to an action on the one-point compactification $X$ of $\overline{X^{\prime}}$ defined by $g \cdot x_{\infty}=x_{\infty}, g \in G$, is as required.

Fix a sequence $\left\{g_{n}\right\}$ of elements of $G$ with $d\left(g_{n}, 1\right) \geq 2 n+1$. Then

$$
d\left(g_{n}^{-1} h, e\right)=d\left(h, g_{n}\right) \geq d\left(g_{n}, e\right)-d(h, e) \geq 2 n+1-n=n+1
$$

for all $h \in \Delta_{n}$. Thus, $g_{n}^{-1} \cdot x_{h} \notin \Delta_{n+1}$. Since such $x_{h}$ form a dense subset of $\Delta_{n}$ and $\Delta_{n} \subseteq \operatorname{Int}\left(\Delta_{n+1}\right)$, it is clear that $g_{n} \cdot \Delta_{n} \cap \Delta_{n}=\emptyset$.

\section{References}

[1] H. Becker and A. Kechris, The Descriptive Set Theory of Polish Group Actions, London Math. Soc. Lecture Note Ser. 232, Cambridge Univ. Press, 1996. 
[2] G. Beer, A Polish topology for the closed subsets of a Polish space, Proc. Amer. Math. Soc. 113 (1991), 1123-1132.

[3] P. J. Cameron, Oligomorphic Permutation Groups, London Math. Soc. Lecture Note Ser. 152, Cambridge Univ. Press, 1990.

[4] J. Dieudonné, Sur la complétion des groupes topologiques, C. R. Acad. Sci. Paris 218 (1944), 774-776.

[5] R. Engelking, Outline of General Topology, North-Holland, 1968.

[6] A. Kechris, Classical Descriptive Set Theory, Grad. Texts in Math. 156, Springer, Berlin, 1995.

[7] —, Lectures on Definable Group Actions and Equivalence Relations, unpublished notes.

[8] E. R. Lorch, Certain compact spaces and their homeomorphism groups, Rend. Sem. Mat. Fis. Milano 52 (1982), 75-86.

[9] L. Newelski and M. Petrykowski, Coverings of groups and types, J. London Math. Soc. (2) 71 (2005), 1-21.

[10] W. Rudin, Real and Complex Analysis, McGraw-Hill, 1986.

[11] S. Solecki, Actions of non-compact and non-locally compact Polish groups, J. Symbolic Logic 65 (2000), 1881-1894.

[12] S. Solecki, Amenability, free subgroups, and Haar null sets in non-locally compact groups, Proc. London Math. Soc. 93 (2006), 693-722.

[13] —, On Haar null sets, Fund. Math. 149 (1996), 205-210.

[14] T. Tsankov, Compactifications of $\mathbb{N}$ and Polishable subgroups of $S_{\infty}$, ibid. 189 (2006), 269-284.

[15] V. V. Uspenskij, On subgroups of minimal topological groups, e-print, 2000, http:// arxiv.org/abs/math/0004119.

Department of Mathematics

University of Illinois at Urbana-Champaign

1409 W. Green St.

Urbana, IL 61801, U.S.A.

E-mail: mamalicki@gmail.com
Current address:

Institute of Mathematics

Polish Academy of Sciences

Śniadeckich 8

00-956 Warszawa, Poland

Received 29 October 2007;

in revised form 19 March 2008 